\title{
DINÂMICA DA TEMPERATURA DA PELE DE EQUINOS DURANTE ATIVIDADE FÍSICA POR MEIO DA TERMOGRAFIA INFRAVERMELHA
}

BIOENG K. de Oliveira ${ }^{1 *}$, G. A. C. Oliveira ${ }^{1}$, D. A. da Silva ${ }^{1}$, L. G. F. Bueno ${ }^{1}$, A. M. Lopes' ${ }^{1}$, D. J. de Moura ${ }^{2}$

${ }^{1}$ UNESP - Universidade Estadual Paulista, Faculdade de Ciências Agrarias e Tecnológicas, Dracena, SP, Brasil

${ }^{2}$ UNICAMP - Universidade estadual de Campinas, Faculdade de Engenharia Agrícola, Campinas, SP, Brasil

Article history: Received 22 August 2018; Received in revised form 25 September 2018; Accepted 12 October 2018; Available online 19 December 2018.

\section{RESUMO}

O objetivo foi descrever as mudanças na temperatura da pele de equinos durante o exercício obtido através da termografia infravermelha, bem como avaliar a funcionamento dos grupos musculares em resposta a atividade física. Foram utilizados dez equinos atletas da raça Quarto de Milha, delineados em parcelas subdivididas, resultando em dez repetições por tratamento. Os tratamentos consistiram das avaliações termográficas da temperatura da pele (Tp) dos cavalos em repouso (MR) e em dois momentos durante o exercício, 5 (M5) e 10 min (M10), totalizando em três tratamentos. Os termogramas obtidos foram analisados para região específica e geral do corpo do cavalo. As regiões específicas foram delineadas com base na anatomia dos equinos, resultando em cinco regiões musculares representando o pescoço, membro torácico, dorso, abdômen e membro pélvico. Verificou-se ausência de efeito significativo $(\mathrm{P}>0,05)$ do exercício sobre a Tp das áreas corporais localizadas no pescoço, membro torácico e dorso. As avaliações realizadas com 5 min de atividade física, comparadas ao MR, resultaram em Tp superior $(\mathrm{P}<0,05)$ para a região abdominal. As Tp monitoradas com 10 min de exercício em relação ao $\mathrm{MR}$ apresentaram aumento $(\mathrm{P}<0,05)$, somente para a região abdominal e pélvica. Concluiu-se que o uso da termografia infravermelha permite identificar com precisão as mudanças na temperatura da pele de equinos em repouso e exercitados. A musculatura abdominal e pélvica foram os principais grupos musculares ativados durante atividade física nos equinos.

Palavras-chave: cavalos, exercício, músculo, termógrafo

\section{DYNAMICS O EQUINE SKIN TEMPERATURE DURING PHYSICAL ACTIVITY BY INFRARED TERMOGRAPHY}

\begin{abstract}
The objective was to describe the changes in the skin temperature of horses during exercise obtained through infrared thermography, as well as to evaluate the functioning of muscle groups in response to physical activity. Ten equine Quarter Horse athletes were used, delineated in subdivided plots, resulting in ten replicates per treatment. The treatments consisted of thermographic evaluations of skin temperature (St) of resting moment (RM)) and at two moments during exercise, 5 (M5) and $10 \mathrm{~min}$ (M10), totaling in three treatments. The obtained thermograms were analyzed for the specific and general region of the horse's body.
\end{abstract}

\footnotetext{
*katia.oliveira@unesp.br
} 
The specific regions resulting in five muscular regions representing the neck, thoracic limb, dorsum, abdomen and pelvic limb. There was no significant effect $(\mathrm{P}>0.05)$ of the exercise on the St of the body areas located in the neck, thoracic limb and back. Evaluations performed with 5 min of physical activity, compared to RM, resulted in superior $\mathrm{Tp}(\mathrm{P}<0.05)$ for the abdominal region. The Tp monitored with $10 \mathrm{~min}$ of exercise compared to RM showed an increase $(\mathrm{P}<0.05)$, only for the abdominal and pelvic region. It was concluded that the use of infrared thermography allows to accurately identify changes in the skin temperature of resting and exercised horses. The abdominal and pelvic muscles were the main muscle groups activated during physical activity in horses.

Keywords: exercise, horses, muscle, thermograph

\section{INTRODUÇÃO}

A pele possui receptores térmicos que são responsáveis por reconhecer diferentes condições ambientais como frio ou calor, que desencadeiam respostas fisiológicas pelo animal de vasoconstrição ou vasodilatação periférica, respectivamente (FERNANDES et al., 2012). Este órgão é fundamental na manutenção da temperatura corporal dos equinos dentro de uma zona de termoneutralidade de 37 à $40^{\circ} \mathrm{C}\left(98,6\right.$ à $\left.40^{\circ} \mathrm{F}\right)$, para preservar as funções vitais do organismo, o que evidencia eficiente capacidade termorregulatória (SIMON et al., 2006). Assim, o animal perde calor por meio de convecção, radiação, evaporação e condução através da pele, bem como pela via respiratória, resultando na evaporação (JODKOWSKA et al., 2011).

A atividade física promove produção de calor, no qual somente de 20 a $35 \%$ da energia é usada pelo músculo para ser convertida em energia mecânica, portanto, há um aumento do calor interno nesta situação (SIMON et al., 2006). Assim, o exercício torna-se um agente perturbador da homeostase térmica, por modificar o equilíbrio térmico do organismo. Isto implica em redistribuição da circulação sanguínea das áreas inativas às ativas durante o exercício. Em seguida, ocorre o redirecionamento do fluxo sanguíneo para a pele, com o objetivo de trocar calor com meio ambiente (FERNANDES et al., 2012). Pesquisas realizadas com humanos (MERLA et al., 2010; ZONTAK et al., 1998), avaliando a temperatura da pele (Tp) durante o exercício com termografia, identificaram a ocorrência deste redirecionamento do fluxo sanguíneo, ou seja, observaram redução na $\mathrm{Tp}$ em indivíduos na fase inicial do exercício (5 $\mathrm{min})$, com incremento da mesma na continuidade da atividade física (a partir de $10 \mathrm{~min}$ ).

A termografia infravermelha (TIR) tem se tornado uma alternativa metodológica emergente nos ensaios que utilizam a $\mathrm{Tp}$ em suas análises, por ser uma técnica não invasiva, sem exposição a radiação (EDDY et al., 2001), com câmera de fácil manuseio, emitindo resultados de forma rápida sem risco de acidentes ao cavalo e ao investigador (WESTERMANN et al., 2013), além do custo reduzido do equipamento atualmente (McCAFFERTY, 2007). Esta câmera detecta a radiação infravermelha (calor) emitida pela superfície corporal e produz imagens termográficas com a distribuição da Tp de regiões específicas (WESTERMANN et al., 2013). Tal observação é importante para estabelecer a forma como os ajustes térmicos da $\mathrm{Tp}$ ocorrem nos equinos durante as diversas atividades físicas. Portanto, o conhecimento da temperatura de superfície da pele em relação a musculatura exercitada poderá se tornar uma ferramenta útil na avaliação de programas de treinamento para equinos atletas.

não tem sido investigada na experimentação de equinos, sendo bem documentado o emprego da termografia nas avaliações de lesões podais, 
articulares, ósseas, tendíneas e musculares dos cavalos (YANMAZ et al., 2007). Neste sentido, objetivou-se descrever as mudanças na temperatura da pele de

\section{MATERIAL E MÉTODOS}

\section{Animais}

O experimento foi conduzido no Haras RR, situado no município de São Pedro/SP. O critério para inclusão de cavalos ao estudo foi idade entre 5 e 10 anos, peso vivo médio de $450 \mathrm{~kg}$, condicionados ao trabalho à guia por dois meses no mínimo, nenhuma claudicação visível e sem sinais ou sintomas compatíveis com lesões musculoesqueléticas. Foram utilizados dez equinos atletas da raça Quarto de Milha, delineados em parcelas subdivididas, resultando em dez repetições por tratamento. Os tratamentos consistiram das avaliações termográficas da Tp dos cavalos em repouso (MR) e em dois momentos durante o exercício, 5 (M5) e $10 \mathrm{~min}$ (M10), totalizando em três tratamentos. Cada tratamento, com dez cavalos, foi avaliado por dia, necessitando-se de três dias para totalizar o período experimental. A atividade física realizada durante as mensurações caracterizou-se no desenvolvimento pelos cavalos de trabalho à guia, em círculo com diâmetro de $18 \mathrm{~m}$, no andamento ao trote e em ritmo médio, no sentido anti-horário, em piso de areia. Todos os cavalos foram alojados individualmente em baia medindo $3 \times 3 \mathrm{~m} \mathrm{e}$ alimentados com concentrado e feno. Ainda, os pelos dos animais foram considerados adequados, apresentando-se fino e curto, concordante com a estação do verão. Nenhum tipo de sedativo foi administrado aos animais durante $\mathrm{o}$ experimento. Este estudo foi certificado pelo Comitê de Ética no Uso de Animais (CEUA), do Curso de Zootecnia, Universidade Estadual Paulista - UNESP, Campus de Dracena, Brasil, sob no. $37 / 2012$, de acordo com o princípio ético da experimentação animal. equinos durante o exercício obtido através da termografia infravermelha, bem como avaliar $\mathrm{o}$ funcionamento dos grupos musculares em resposta a atividade física.

\section{Procedimento Experimental}

As imagens termográficas foram realizadas com câmera infravermelha portátil (Testo ${ }^{\circledR} 880$ ), com acurácia de \pm $0,1^{\circ} \mathrm{C}$ e intervalo de espectro infravermelho de 7,5 à $13 \mathrm{mM}$. O índice de emissividade utilizado foi de 0,95 (AUTIO et al., 2006). A temperatura do ambiente foi anotada diariamente, mediante uso de termômetro, variando de 30 a $33^{\circ} \mathrm{C}$, possibilitando a correção dos dados de Tp dos cavalos coletados nos diferentes dias, de acordo com Basile et al. 2010. A velocidade do vento foi considerada nula, devido ao procedimento de colheita das imagens termográficas terem ocorrido em pista coberta, do tipo indoor. Previamente a aquisição das imagens termográficas $(1 \mathrm{~h}$ antes) os cavalos foram escovados para remoção de sujeiras dos pelos e mantidos secos de qualquer tipo de umidade, chuva ou suor. Procedeu-se a estabilização dos cavalos ao ambiente de avaliação por tempo de 40 min (TUNLEY \& HENSON, 2004). As imagens foram realizadas a uma distância do cavalo de $4 \mathrm{~m}$, perpendicularmente a lateral esquerda do mesmo (SIMON et al., 2008). Durante a captação das imagens os cavalos foram contidos por meio de cabresto com cabo de corda, na pista indoor. As imagens foram analisadas no próprio software da câmera (Testo IRSoft Software), com a escala definida na opção de paleta frio/quente e com a temperatura variando entre $17 \mathrm{e}$ $40^{\circ} \mathrm{C}$.

\section{Variáveis Avaliadas}

Os termogramas obtidos foram analisados para região específica e geral do corpo do cavalo. As regiões específicas foram delineadas com base na anatomia dos equinos, resultando em cinco regiões musculares representando o pescoço, membro torácico, dorso, abdômen e 
membro pélvico, conforme esquema apresentado na (Figura 1). Foi determinada a Tp média e máxima, para caracterizar a dinâmica da temperatura de superfície da pele durante o exercício e para avaliar o funcionamento dos grupos musculares em resposta ao exercício (JODKOSKA et al, 2011), respectivamente. As $\mathrm{Tp}$ média e máxima foram calculadas a partir de um polígono delineado para cada região estudada mediante uso do Testo IRSoft Software. Ainda, determinaram-se as mudanças de $\mathrm{Tp}$ entre as imagens em repouso com relação àquelas obtidas durante o exercício (WESTERMANN et al., 2013).
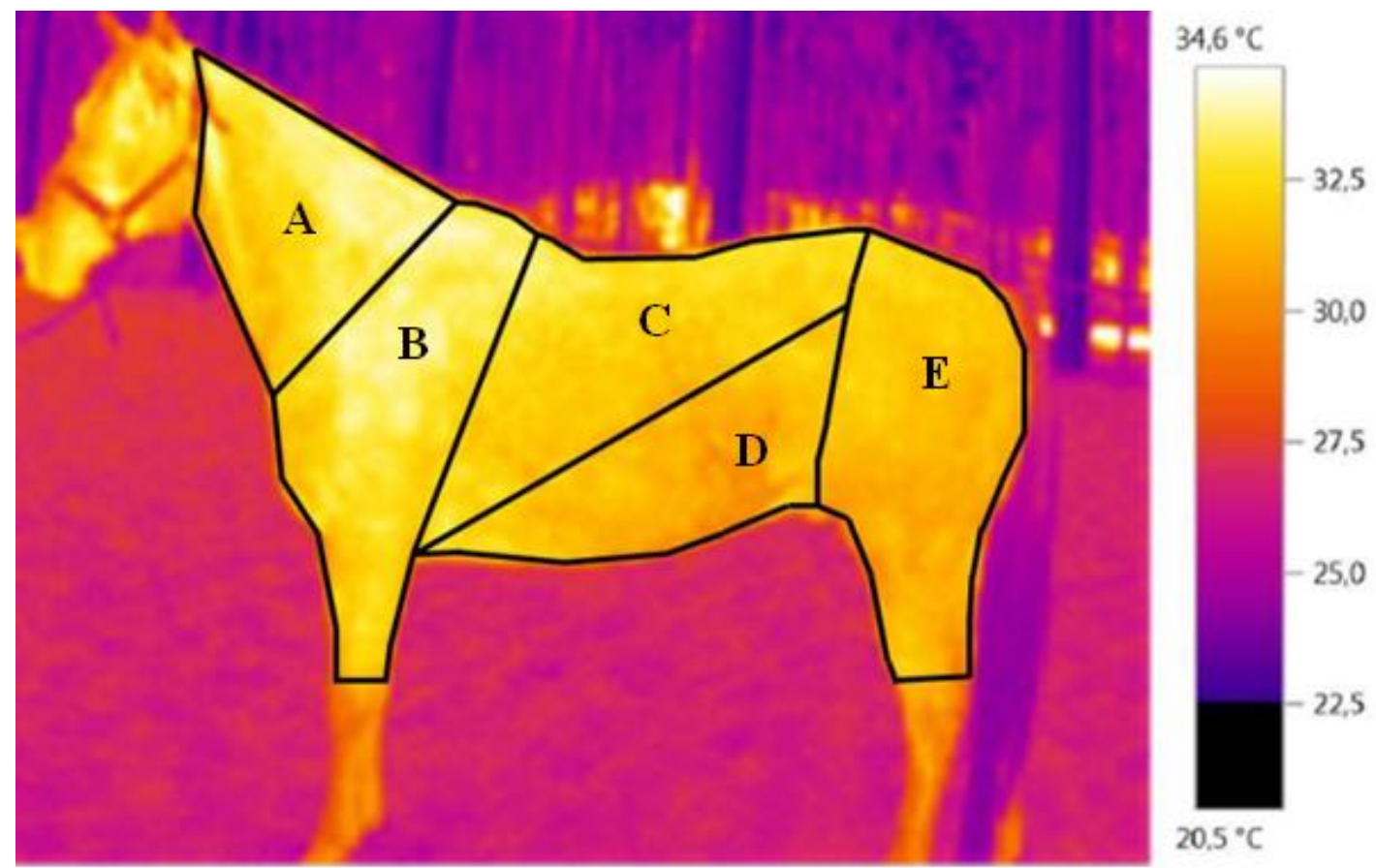

Figura 1: Ilustração da divisão das regiões musculares para a determinação da temperatura da pele nos equinos: pescoço (A), membro torácico (B), dorso (C), abdômen (D) e membro pélvico (E).

\section{Análise estatística}

As variáveis foram avaliadas quanto à normalidade de distribuição, usando o teste Kolmogorov-Smirnov e as variáveis não distribuídas normalmente foram transformadas em log. O coeficiente de variação foi usado para expressar a quantidade de variabilidade nas variáveis.

\section{RESULTADOS E DISCUSSÃO}

Na Tabela 1, encontram-se os resultados da temperatura da pele das
A dinâmica das temperaturas das superfícies corporais dos cavalos e o funcionamento dos grupos musculares foram submetidos à análise de variância do Statistical Analysis System (SAS, 2000) e as comparações entre as médias por meio do teste de Tukey ao nível de significância de $5 \%$.

regiões corporais de equinos em repouso e durante o exercício. 
Tabela 1 - Valores da temperatura da pele média $\left({ }^{\circ} \mathrm{C}\right)$ das regiões corporais de cavalos avaliados durante os momentos de repouso (MR) e em exercício

\begin{tabular}{lccccc}
\hline \multirow{2}{*}{ Regiões corporais } & \multicolumn{3}{c}{ Momento de avaliação } & \multirow{2}{*}{ Valor de P* } & $\begin{array}{c}\text { Coeficiente de } \\
\text { Variação (\%) }\end{array}$ \\
\cline { 2 - 4 } & MR & M $^{1}$ & M10 & & 3,92 \\
\hline Pescoço & 32,95 & 33,87 & 33,59 & 0,2643 & 4,24 \\
Membro Torácico & 32,77 & 33,85 & 33,72 & 0,1385 & 4,37 \\
Dorso & 32,67 & 33,93 & 33,73 & 0,0786 & 4,47 \\
Abdômen & $32,33^{\mathrm{b}}$ & $33,93^{\mathrm{a}}$ & $34,24^{\mathrm{a}}$ & 0,0012 & 4,59 \\
Membro Pélvico & $32,20^{\mathrm{b}}$ & $33,68^{\mathrm{ab}}$ & $34,03^{\mathrm{a}}$ & 0,0038 & 4,22 \\
Geral & 32,58 & 33,85 & 33,87 & 0,0333 & \\
\hline
\end{tabular}

${ }^{1} \mathrm{M} 5=$ momento de avaliação com 5 min de exercício; ${ }^{2} \mathrm{M} 10=$ momento de avaliação com 10 min de exercício; *Médias seguidas por letras diferentes, na linha, diferem entre si $(\mathrm{P}<0,05)$ pelo teste de Tukey.

Verificou-se ausência de efeito significativo $(\mathrm{P}>0,05)$ do exercício sobre a $\mathrm{Tp}$ das áreas corporais localizadas no pescoço, membro torácico e dorso, em que os valores médios foram de $33,47,33,45$ e $33,44{ }^{\circ} \mathrm{C}$, respectivamente. Tal resposta pode estar associada a baixa capacidade vasomotora destas regiões, apesar do aumento de fluxo sanguíneo em função do exercício, bem como pela menor ação da musculatura para realização da atividade física, já que o exercício aumenta a produção de calor corporal (McCUTCHEON \& GEOR, 2008).

As avaliações realizadas com $5 \mathrm{~min}$ de atividade física, comparadas ao momento de repouso, resultaram em $\mathrm{Tp}$ iguais $(\mathrm{P}>0,05)$, para o pescoço, membro torácico, dorso e membro pélvico, e superior $(\mathrm{P}<0,05)$ para a região abdominal. Este comportamento de resposta em equinos, se diferencia ao encontrado nas pesquisas realizadas com humanos (MERLA et al., 2010; ZONTAK et al., 1998), no qual observaram redução na Tp em indivíduos na fase inicial do exercício (5 min). Portanto, não sendo possível observar no atual ensaio com cavalos, o

\section{CONCLUSÕES}

O uso da termografia infravermelha permitiu identificar com precisão as mudanças na temperatura da pele de equinos em repouso e exercitados. fenômeno de redistribuição da circulação sanguínea das áreas inativas às ativas durante o exercício, que culmina com a redução da $\mathrm{Tp}$ nos primeiros $5 \mathrm{~min}$ do exercício em humanos (FERNANDES et al., 2012).

As Tp monitoradas com $10 \mathrm{~min}$ de exercício em relação ao momento de repouso apresentaram aumento $(\mathrm{P}<0,05)$, somente para a região abdominal e pélvica, no valor de 1,91 e $1,83^{\circ} \mathrm{C}$, respectivamente (Tabela 1). Esta comparação foi realizada para rastrear as áreas musculares mais ativadas durante atividade física, por meio da $\mathrm{Tp}$, mediante uso da termografia infravermelha. Os resultados encontrados da presente pesquisa atestam a eficácia desta técnica na identificação dos músculos ativados durante atividade física, pois são concordantes aos resultados de Sala et al. (2012). Neste trabalho, os pesquisadores mensuraram em cavalos da raça Quarto de milha, a Tp em situação de esforço físico, no qual resultou em incremento na $\mathrm{Tp}$ da musculatura pélvica, mais especificamente do músculo semimembranoso, seguido pelo semitendinoso e do glúteo superior.

A musculatura abdominal e pélvica foram os principais grupos musculares ativados durante atividade física nos equinos. 


\section{REFERÊNCIAS BIBLIOGRÁFICAS}

EDDY, A.L.; VAN HOOGMOED, L.M.; SNYDER, J.R. The Role of Thermography in the Management of Equine Lameness. The Veterinary Journal, v.162, p.172181, 2001.

FERNADES, A.A.; AMORIM, P.R.S.; PRIMOLA-GOMES, T.N.; SILLEROQUINTANA, M.; FERNÁNDEZ CUEVAS, I.; SILVA, R.G.; PEREIRA, J.C.; MARINS, J.C.B. Avaliação da temperatura da pele durante o exercício através da termografia infravermelha: uma revisão sistemática. Revista Andaluza de Medicina del Deporte, v.5, p.113-117, 2012.

JODKOWSKA, E.; DUDEK, K.; PRZEWOZNY, M. The Maximum Temperatures (Tmax) Distribuition on the Body Surface of Sport Horse. Journal of Life Sciences, v.5, p.291-297, 2011.

McCAFFERTY, D.J. The value of infrared thermography for research on mammals: previous applications and future directions. Mammal Review, v.37, p.207-223, 2007.

McCUTCHEON, 1.j.; GEOR, R.J. Redistribution of cardiac output in response to heat exposure in the pony. Equine Veterinary Journal, v.22, p.4246, 2008.

MERLA, A.; MATTEI, P.A.; DI DONATO, L.; POMANI, G.L. Thermal imaging of cutaneous temperature modifications in runners during graded exercise. Ann Biomedical Engineering, v.38, p.158-163, 2010.

SALA, L.C.C.; ELUI, M.C.; JARDIM, M.C. Avaliação termográfica da musculatura pélvica de equinos da modalidade esportiva de três tambores.
Publicações em Medicina Veterinária e Zootecnia, v.6, n.29, p.1437, 2012.

SIMON, E.L.; GAUGHAN, E.M.; EPP, T.; SPIRE, M. Influence of exercise on thermographically determined surface temperatures of thoracic and pelvic limbs in horses. Journal of the American Veterinary Medical Association, v.229, p.1940-1944, 2006.

STATISTICAL ANALYSIS SISTEM SAS. SAS user's: guide statistics. Cary: 2000. 211p.

TUNLEY, B.V.; HERSON, F.M.D. Reliability and repeatability of thermographic examination and the normal thermographic image of the thoracolumbar region in the horse. Equine Veterinary Journal, v.36, p.306-312, 2004.

WESTERMANN, S.; BUCHNER, H.H.F.; SCHRAMEL, J.P.; TICHY A.; STANEK, C. Effects of infrared camera angle and distance on measurement and reproducibility of thermographically determined temperatures of the distolateral aspects of the forelimbs in horses. Journal of the American Veterinary Medical Association, v.242, p.388-395, 2013.

YANMAZ, L.E.; OKUMUS, Z.; DOGAN, E. Instrumentation of Thermography and its Applications in Horses. Journal of Animal and Veterinary Advances, v.6, p.858-862, 2007.

ZONTAK, A.; SIDEMAN, S.; VERBITSKY, O.; BEYAR, R. Dynamic thermography: Analysis of Hand temperature During Exercise. Annals of Biomedical Engineering, v.26, p.988-993, 1998. 